\title{
SELECCIÓN DE UNA LEVADURA PARA LA PRODUCCIÓN DE BIOMASA: CRECIMIENTO EN SUERO DE QUESO ${ }^{1}$
}

\author{
Wendy Zumbado-Rivera ${ }^{2}$, Patricia Esquivel-Rodríguez ${ }^{2}$, Eric Wong-González ${ }^{2}$
}

\begin{abstract}
RESUMEN
Selección de una levadura para la producción de biomasa: crecimiento en suero de queso. El presente proyecto se realizó con el objetivo de recomendar una especie de levadura para la producción de biomasa, utilizando como sustrato el suero de leche del proceso de elaboración de queso blanco tipo Turrialba. Se compararon las especies Kluyveromyces marxianus, Candida kefyr y Saccharomyces cerevisiae por medio de su crecimiento en un sistema de fermentación por lotes. Se determinó el tiempo de fermentación, la productividad total y el contenido de proteína de la biomasa. Al comparar la variación en el tiempo según la levadura utilizada, se obtuvo diferencias significativas $(\mathrm{p} \leq$ 0,05). Las levaduras $K$. marxianus y $C$. kefyr presentaron un tiempo de 19 y 18 horas, respectivamente y $S$. cerevisiae, un tiempo de 24 horas. Con respecto a la productividad total de biomasa, la especie $K$. marxianus presentó un valor de 0,22 $\mathrm{g} / \mathrm{lxh}$, resultando significativamente diferente $(\mathrm{p} \leq 0,05)$ a las especies $C$. kefyr y $S$. cerevisiae, para las cuales se obtuvieron productividades de 0,14 y $0,13 \mathrm{~g} / \mathrm{lxh}$. La variación en el contenido de proteína de acuerdo con la levadura utilizada no resultó significativo ( $\mathrm{p} \geq 0,05$ ). Para la especie $K$. marxianus se obtuvo un contenido de proteína de 32\%, 34\% para C. kefyr y $35 \%$ para $S$. cerevisiae. Se seleccionó la especie de levadura Kluyveromyces marxianus para la producción de proteína unicelular, por presentar un menor tiempo de fermentación, mayor productividad e igual contenido proteico de la biomasa que las otras levaduras, además de facilidades de utilización.
\end{abstract}

Palabras clave: Proteína unicelular, lactosa, Saccharomyces cerevisiae, Candida kefyr, Kluyveromyces marxianus.

\begin{abstract}
Selection of yeast for biomass production based on the cultivation results on whey. The objective of this research was to recommend a yeast species useful for biomass production in the form of single cell protein, using whey obtained form Turrialba cheese manufacturers as substrate. Three yeasts (Kluyveromyces marxianus, Candida kefyr and Saccharomyces cerevisiae) were grown separately in a batch fermentation system, and the fermentation time, total productivity and protein content of the biomass were determined. Significant differences $(\mathrm{p}<0.05)$ were found in the fermentation times required by each yeast. K. marxianus y $C$. kefyr respectively showed a fermentation time of 19 and $18 \mathrm{~h}$, while $S$. cerevisiae needed 24 h. $K$ marxianus showed a productivity of $0.22 \mathrm{~g} / \mathrm{Lxh}$, significantly different $(\mathrm{p}<0.05)$ from the productivities of $C$. kefyr and $S$. cerevisiae, with values of $0.14 \mathrm{~g} / \mathrm{Lxh}$ and 0.13 $\mathrm{g} / \mathrm{Lxh}$, respectively. The protein content of the biomass for C. kefyr, S. cerevisiae, and K. marxianus were respectively of $34 \%, 35 \%$ and $32 \%$. Considering the lowest time required for fermentation, the highest productivity and an equal content of protein in the biomass when compared to the other two yeasts, Kluyveromyces marxianus was chosen as the recommended yeast to produce single cell protein from cheese whey.
\end{abstract}

Key words: Single cell protein, lactose, Saccharomyces cerevisiae, Candida kefyr, Kluyveromyces marxianus.

\footnotetext{
1 Recibido: 7 de setiembre, 2005. Aceptado: 17 de julio, 2006. Proyecto de graduación para optar por el grado de Licenciatura en Tecnología de Alimentos. Universidad de Costa Rica.

2 Escuela de Tecnología de Alimentos, Universidad de Costa Rica. San Pedro, San José, Costa Rica. Correo electrónico: wzumbado@gmail.com.
} 


\section{INTRODUCCIÓN}

El suero de leche es el principal subproducto en la fabricación de quesos. Según datos reportados, en el 2001 se generaron aproximadamente 332 millones de litros de suero de leche (CNP 2001). En Costa Rica, la utilización del suero de leche no se ha industrializado, su aprovechamiento está limitado por la dificultad de transporte y porque al igual que el resto de productos lácteos, es perecedero, lo que hace imposible almacenarlo, sin ningún tratamiento, por más de dos días (Barroquio et al. 1981). Si el suero es desechado en los ríos se convierte en una grave contaminación, pues presenta una demanda biológica de oxígeno $\left(\mathrm{DBO}_{5,20}\right)$ muy alta, que se encuentra entre los 40.000 y los $50.000 \mathrm{mg} \mathrm{O}_{2} / 1$ (Salazar 1999).

Al generarse elevados volúmenes de suero de leche, y con niveles de contaminación superiores a la cantidad máxima permitida para el vertido de aguas residuales en ríos o alcantarillado público (300 $\left.\mathrm{mg} \mathrm{O}_{2} / \mathrm{l}\right)$ (Ministerio de Salud 1997), resulta necesario buscar métodos para su aprovechamiento. Una alternativa es utilizar el suero como medio para el crecimiento de microorganismos para la producción de biomasa. La masa de microorganismos obtenida (levaduras, mohos, bacterias, algas) posee un alto contenido proteico, por lo que recibe el nombre de proteína unicelular. En diversas investigaciones se ha indicado la posibilidad de obtener proteína unicelular mediante el crecimiento de microorganismos que hacen uso de la lactosa presente en el suero (Bu'Lock y Kristiansen 1991; Hernández et al. 1980; Grba et al. 2002). Especies de levadura como Kluyveromyces marxiaus y Candida kefyr son capaces de fermentar la lactosa, al ser productoras de la enzima B-D-Galactosidasa (EC 3.2.1.23), la cual hidroliza la lactosa en sus componentes básicos (Rajoka et al. 2003; Inchaurrondo et al. 1994). Ambas levaduras han sido empleadas en investigaciones para la generación de biomasa, etanol y para la producción de la enzima (Belem et al. 1997).

Para la generación de biomasa a partir de la lactosa del suero también es posible el empleo de levaduras de la especie Saccharomyces cerevisiae, cuyo crecimiento se logra si la lactosa es hidrolizada previamente en glucosa y galactosa, por medio de preparados enzimáticos (Mittal 1992). Esta especie de levadura presenta una amplia utilización y aceptación en la industria para la alimentación animal y humana, lo que podría resultar en una ventaja para su utilización (Liti et al. 2001).

El proceso de producción de proteína unicelular es una vía biotecnológica adecuada para el aprovechamiento de desechos industriales ricos en carbohidratos. De hecho, el principal factor limitante en la generación de proteína unicelular es el alto costo de las fuentes de carbono, por lo que el uso de subproductos es ideal (Durán 1989). El emplear el suero en este proceso permitiría darle valor agregado, al obtenerse proteína de calidad para la alimentación animal, y a la vez disminuir su DBO para su posterior descarga (Ghaly y Kamal 2004). Esta disminución, según datos reportados para levaduras, se encuentra entre $70-80 \%$ del DBO inicial del suero, lo que convierte este proceso en una opción adecuada para su tratamiento (Capoor y Singh 1985; Chinappi y Sanchez 2000).

El interés por producir proteína unicelular fue estimulado por agencias internacionales relacionadas con la salud, alimentación y agricultura ante la evidencia de una escasez de proteína a nivel mundial (BuLock y Kristiansen 1991), es por esto que la búsqueda de fuentes proteicas es vital (Chacón 2004).

La biomasa de origen unicelular tiene aplicaciones como suplemento proteico en alimentación animal, además, se ha investigado su utilización en la fabricación de ingredientes funcionales, suplementos proteicos, para resaltar el sabor de alimentos procesados, entre otros (Lee 1996). Para su aplicación en la alimentación humana, requiere el empleo de métodos para la reducción del nivel de ácidos nucleicos, ya que si son consumidos en altas proporciones podrían causar la formación de cálculos renales u otras enfermedades. A diferencia de los humanos, el ganado tolera altos niveles de ácidos nucleicos, por lo que la proteína unicelular podría utilizarse inicialmente en alimentación animal sin requerir ningún tratamiento (Lee 1996).

Los componentes del suero que tienen mayor importancia para ser utilizados como sustrato son lactosa, proteína y minerales (Quintero et al. 2001). Con el fin de lograr una utilización eficiente de estas sustancias, es importante elegir una levadura con adecuadas características fisiológicas (Grba et al. 2002). Por lo anterior, considerando la evidencia científica de las características de las levaduras Kluyveromyces marxianus, Candida kefyr y Saccharomyces cerevisiae para su 
desarrollo en el suero de queso, así como su utilización en procesos biotecnológicos (Inchaurrondo et al. 1994; Quintero et al. 2001; Rose 1979; Moo-Young et al. 1985), el objetivo de este proyecto es evaluar el crecimiento de tres especies de levaduras en el proceso de producción de proteína unicelular, con el fin de seleccionar y recomendar una de ellas para ser utilizada en el suero de queso. Uno de los criterios de selección del microorganismo a emplear en el proceso es la productividad de biomasa, unido a otros resultados como son el tiempo de fermentación, el contenido de proteína de la biomasa y aspectos económicos, los cuales se derivarían de características propias de la utilización de cada levadura, como pretratamientos del sustrato.

El producto obtenido se podría destinar a la alimentación animal y en el futuro continuar las investigaciones para utilizarlo en la alimentación humana.

\section{MATERIALES Y MÉTODOS}

La presente investigación se llevó a cabo de abril del 2004 a marzo del 2005 en las instalaciones de la Universidad de Costa Rica, en San Pedro de Montes de Oca, San José, Costa Rica.

\section{Suero de queso utilizado}

Se utilizó el suero residual de la elaboración de queso blanco tipo Turrialba de una empresa productora de Cartago. Este tipo de queso corresponde la categoría de quesos frescos que se elaboran de cuajada sin maduración. La tradición latinoamericana se basa en quesos blancos elaborados a partir de leche de vaca. El proceso de elaboración parte de leche parcialmente descremada o leche entera. Se procede a su coagulación utilizando cultivo, cuajo y ácido, y el producto obtenido luego del desuerado es salado, moldeado, posteriormente prensado y es consumido en su estado fresco (Revilla 1985).

El queso blanco tipo Turrialba es de color blanco amarillento, de sabor simple, no ácido, de textura suave y de corte limpio y no grasoso. Presenta un elevado contenido de humedad, mayor del 50\%, un contenido de grasa que varía entre $10-26 \%$ y un contenido de sal que varía entre 1-3\% (CNP 1999).
Las muestras se tomaron directamente de la tina de desuerado y fueron transportados en recipientes higienizados de aluminio con tapa, los cuales fueron previamente lavados y desinfectados.

\section{Levaduras y condiciones de mantenimiento}

a. Kluyveromyces marxianus sp. marxianus LAF-4, liofilizado. Almacenado a una temperatura de $-40{ }^{\circ} \mathrm{C}$. Casa comercial Chr. Hansen. Levadura lactosa positivo.

b. Candida kefyr ATCC (2004) 66028, liofilizado, lactosa positivo. Esta fue mantenida en el medio Saborou glucosado (Difco, Becton, Dickinson and Company) a temperatura ambiente.

c. Saccharomyces cerevisiae, levadura seca instantánea de la casa comercial Fleishmann. Almacenado a una temperatura de $4^{\circ} \mathrm{C}$. Levadura lactosa negativo y glucosa positivo.

\section{Equipo de fermentación}

Se utilizó un fermentador diseñado para el estudio (Figura 1), consta de un frasco de fermentación (capacidad de 21 ), unidades de toma de muestra, inoculación y salida de gases. El mantenimiento de la temperatura se realizó empleando una incubadora que se ajustó a la temperatura del proceso. Para la aireación se utilizó una bomba pequeña de 2,5 1/min de capacidad, conectada a un rotámetro, por medio del cual se reguló el flujo de aire. El aire fue esterilizado haciéndolo pasar previamente a través de un filtro de $0,22 \mu \mathrm{m}$.

Para la agitación del medio se utilizó una pastilla de agitación magnética colocada dentro del frasco de fermentación.

\section{Proceso de fermentación}

\section{a. Preparación del suero de leche}

Se llevó a cabo la preparación de la totalidad del suero de queso a emplearse para el crecimiento de las tres levaduras. 


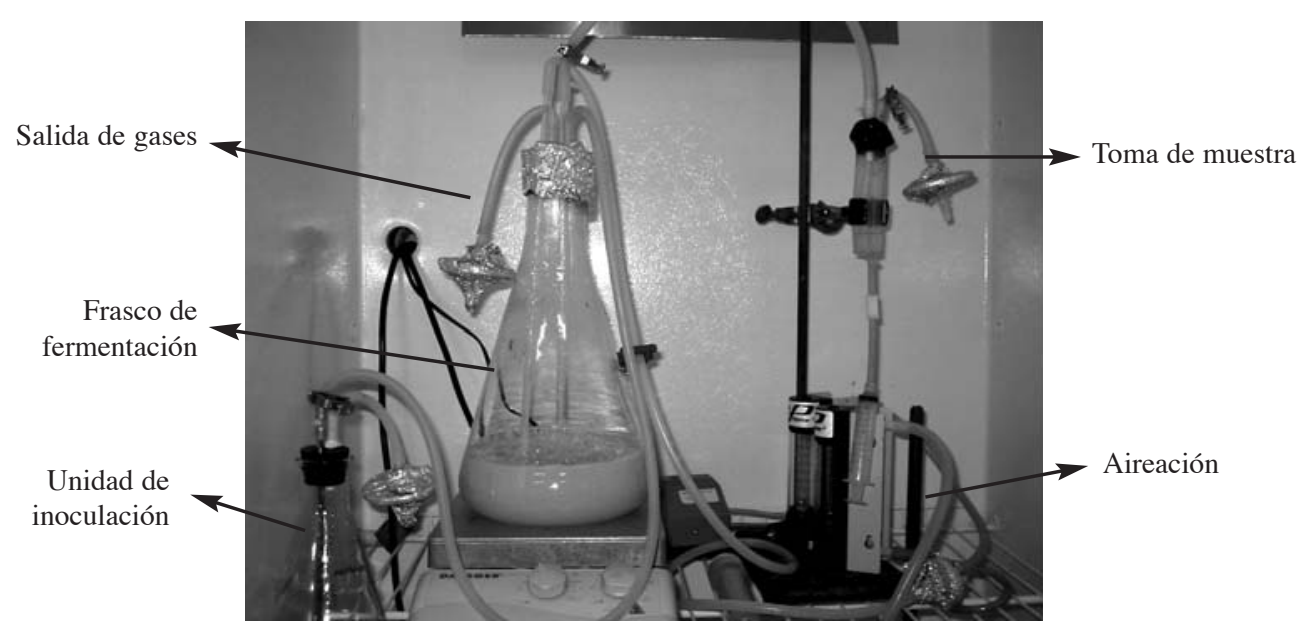

Figura 1. Esquema del equipo de fermentación utilizado para la selección del microorganismo a utilizar en la producción de proteína unicelular. Costa Rica, abril 2004-marzo 2005.

Se separaron las proteínas coagulables del suero de leche por medio de un tratamiento termoácido, en el que se ajustó el pH a 4,5 $(\mathrm{HCl} 1 \mathrm{~N})$ seguido de un calentamiento a $115^{\circ} \mathrm{C}$ por 20 minutos en autoclave (Yamato, Scientific America Inc, Orangeburg). El suero se enfrió y se separaron las proteínas por decantación y filtrado con papel Whatman $\mathrm{N}^{\circ} 41$. El contenido de proteína final del suero empleado en las fermentaciones fue de $0,38 \%$.

Para la preparación del suero de queso para el crecimiento de Saccharomyces cerevisiae se tomó el suero desproteinizado y posteriormente se llevó a cabo la hidrólisis de la lactosa empleando un preparado enzimático de lactasa Maxilact $2000 \circledR$, con lo que se obtuvo suero hidrolizado con un contenido de glucosa de $20 \mathrm{~g} / \mathrm{l}$.

\section{b. Preparación del inóculo}

Cada una de las levaduras estudiadas se inoculó en un Erlenmeyer con $100 \mathrm{ml}$ de caldo Saborou glucosado (Difco, Becton, Dickinson and Company) y se incubó a una temperatura de $30^{\circ} \mathrm{C}$. Kluyveromyces marxianus y Candida kefyr, se incubaron durante 24 horas y Saccharomyces cerevisiae durante cinco horas, previas a la fermentación. La concentración celular se midió por medio de una cámara de Neubauer (Counting Chamber, Hausser Scientific Company) y se estableció en $10^{7}$ células/ml para las tres levaduras.

\section{c. Esterilización del equipo e inoculación}

Se esterilizó un volumen de $900 \mathrm{ml}$ de suero de leche en el frasco de fermentación, junto con las mangueras y filtros acoplados. Se aplicó una temperatura de $115^{\circ} \mathrm{C}$ durante 20 minutos, empleando una autoclave (Yamato Scientific America Inc, Orangeburg). El equipo se enfrió a la temperatura de fermentación y se realizó la inoculación de la levadura.

Los parámetros que se mantuvieron constantes durante el proceso de fermentación con las tres levaduras, fueron temperatura de $30^{\circ} \mathrm{C}$, flujo de aireación 0,6 VVM y agitación $300 \mathrm{rpm}$.

\section{Determinación de la composición del suero}

Se analizaron muestras de suero de queso de 10 lotes distintos escogidos aleatoriamente. A cada muestra se le determinó la cantidad de lactosa, proteína, y humedad utilizando el equipo Milkoscan (Foss Instruments). Además, se realizó la medición del pH por el método 981.12 de la AOAC (1999) y la determinación del contenido de cenizas, por el método 930.30 de la AOAC (1999). Para cada uno de los componentes se reportó el promedio y su correspondiente intervalo de confianza al $95 \%$. 


\section{Determinación del tiempo de fermentación}

Para cada levadura se llevaron a cabo tres repeticiones del proceso de fermentación descrito anteriormente, para cada repetición se construyó una curva de crecimiento, en la que se representó la variación de absorbancia en el tiempo (Scragg 2000). La medición de la absorbancia se realizó en un espectrofotómetro a 620 $\mathrm{nm}$, a muestras de $10 \mathrm{ml}$ del medio que fueron extraídas del biorreactor durante períodos de tiempo establecidos.

El tiempo de fermentación se obtuvo gráficamente en la curva de crecimiento, mediante el trazo de una curva de mejor ajuste del tipo media móvil utilizando el programa Microsoft Excel ${ }^{\mathrm{TM}}$. En el primer punto en el que la curva de mejor ajuste se volvía constante se realizó la interpolación del tiempo de fermentación correspondiente.

\section{Determinación de la productividad de biomasa de las levaduras}

Para cada una de las levaduras se realizaron tres repeticiones del proceso de fermentación. Una vez alcanzada la fase estacionaria se tomaron muestras de $25 \mathrm{ml}$ de la biomasa para la determinación del peso seco. La muestra se centrifugó a una velocidad de 3000 rpm por $30 \mathrm{~min}$, se resuspendió en agua y se sometió a secado a $100^{\circ} \mathrm{C}$ en placas de Petri prepesadas hasta llegar a peso constante. Posteriormente, se calculó la productividad expresada como la relación entre el peso seco de biomasa y el tiempo de fermentación.

\section{Determinación del contenido de proteína de la bio- masa obtenida}

La biomasa seca se sometió a un análisis de la cantidad de proteína por el método de Kjeldahl 930.29 de la AOAC (2000). La medición se llevó a cabo para cada una de las repeticiones del experimento y con cada una de las levaduras.

\section{Diseño experimental y análisis estadístico}

Para el análisis del tiempo de fermentación, la productividad y el contenido de proteína obtenido con cada una de las levaduras se utilizó un diseño irrestricto al azar con tres tratamientos, representados por las tres levaduras. Este se llevó a cabo para las tres variables por separado.

Se realizó un análisis de varianza para evaluar las diferencias en los resultados de cada una de uno de los parámetros según la levadura utilizada. En los casos que se obtuvo diferencia significativa entre los tratamientos ( $\mathrm{p} \leq 0,05)$, se llevó a cabo una prueba de Tukey, de comparación múltiple de promedios, para determinar con cuál levadura se obtuvo un efecto distinto.

\section{RESULTADOS Y DISCUSIÓN}

\section{Composición del suero de queso}

En el Cuadro 1 se presenta la caracterización del suero empleado en la investigación.

Cuadro 1. Composición promedio del suero de queso utilizado para la producción de proteína unicelular. Costa Rica, abril 2004-marzo 2005.

\begin{tabular}{lcc}
\hline Componente & $\begin{array}{c}\text { Contenido } \\
(\boldsymbol{\%} \mathbf{~ m} / \mathbf{m})\end{array}$ & $\begin{array}{c}\text { Intervalo de } \\
\text { confianza } \mathbf{( \pm )}\end{array}$ \\
\hline Lactosa & $4,77^{*}$ & 0,08 \\
Proteína & $0,96^{*}$ & 0,02 \\
Cenizas & $0,52^{*}$ & 0,01 \\
Humedad & $93,10^{*}$ & 0,20 \\
pH & 6,54 & 0,03 \\
\hline
\end{tabular}

* Existieron diferencias significativas entre datos que originaron los valores promedio ( $\mathrm{p} \leq 0,05)$.

El suero utilizado presentó diferencias significativas entre lotes en cuanto al contenido de sus componentes más importantes. Esto podría generar resultados de fermentación distintos y el requerir la estandarización del sustrato para la aplicación del proceso a nivel industrial.

Con el fin de reducir el efecto del sustrato, se realizó la preparación de la totalidad del suero de queso a emplearse para el crecimiento de las levaduras en estudio.

El suero analizado presentó como componente mayoritario el agua, seguido por la lactosa, proteína y cenizas. Un contenido de $4,77 \%$ de lactosa representa 
una concentración adecuada para el crecimiento de levaduras, según resultados de diversas investigaciones en las que se han utilizado concentraciones similares (Chinappi y Sánchez 2000; Quintero et al. 2001; Ghaly y Kamal 2004). El nivel de azúcar presente es una de las razones que convierte al suero en un sustrato óptimo para la generación de biomasa (BúLock y Kristiansen 1991; Lee 1996; Ward 1991).

El porcentaje de proteína en el suero es similar al presentado en investigaciones realizadas con distintos tipos de suero (Chinappi y Sánchez 2000; Quintero et al. 2001). Se ha observado que el utilizar suero sin la adición de otras fuentes de nitrógeno permite obtener biomasa microbiana con contenidos de proteína mayores al 40\% (Rose 1979). En el presente estudio se utilizó el suero sin suplementar con el fin de evaluar el crecimiento de las levaduras en un medio simple.

El contenido de cenizas concuerda con la composición promedio reportada para el suero de leche (Alais 1996). Según Chinnapi y Sánchez (2000) y Moo-Young et al. (1985), el contenido de minerales del suero permite el crecimiento adecuado de levaduras (Rose 1979).

De acuerdo con el pH del suero, éste se clasifica como suero dulce, al presentar un valor mayor a 5,9, que es el esperado a partir de elaboración de quesos blancos (Revilla 1985). Dado que su pH es alto y que presenta un alto contenido de humedad, el pH del suero se debe ajustar a un valor de 4,5 al inicio de la fermentación, con el fin de reducir la posibilidad de contaminación con bacterias (Moo-Young et al. 1985).

\section{Comparación del tiempo de fermentación}

En la Figura 2, donde se presentan las curvas de crecimiento promedio de las levaduras, se puede distinguir la fase lag o de adaptación de los microorganismos al medio. También se observa el momento en el que se llega a la fase estacionaria, donde no se observan cambios aparentes en la absorbancia con respecto al tiempo, lo que indica que no se está generando más masa celular (Chan 2001).

A partir de las curvas de crecimiento, es posible observar que la fase de adaptación fue más extensa para las levaduras $K$. marxianus y $C$. kefyr, que a

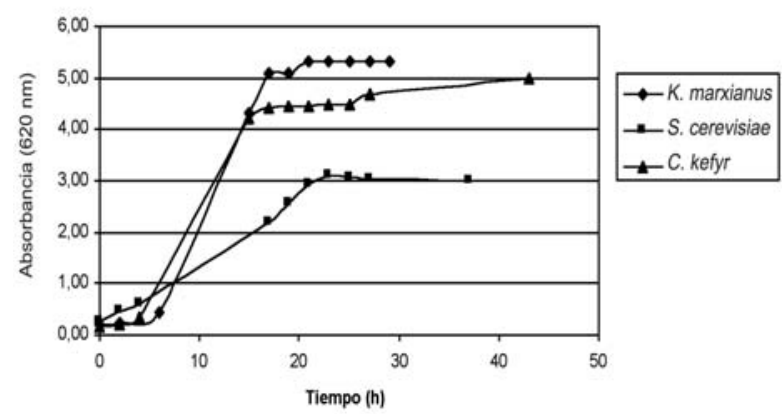

Figura 2. Curva de crecimiento de Kluyveromyces marxianus, Saccharomyces. cerevisiae y Candida kefyr en suero de queso, utilizada en la determinación del tiempo en que se alcanza la fase estacionaria. Costa Rica, abril 2004-marzo 2005.

diferencia de $S$. cerevisiae, debían producir la enzima lactasa para hacer uso de la lactosa del medio (Liti et al. 2001). La modificación del sistema enzimático para iniciar la síntesis de enzimas (Shuler y Kargi 1992) pudo causar que se observara más prolongada.

En el Cuadro 2 se observa que $S$. cerevisiae presentó un tiempo más prolongado para alcanzar la fase estacionaria $(\mathrm{p} \leq 0,05)$ que el de $K$. marxianus y $C$. $k e f y r$, las cuales requirieron cinco y seis horas menos, respectivamente.

En el caso de $S$. cerevisiae, el requerir un tiempo de fermentación mayor puede deberse a características propias de este microorganismo y/o a que su crecimiento en el suero se dio de forma más lenta. En un sistema de producción por lote, un tiempo de fermentación más

Cuadro 2. Tiempo para alcanzar la fase estacionaria en la curva de crecimiento en suero de queso, para Kluyveromyces marxianus, Saccharomyces cerevisiae y Candida Kefyr. Costa Rica, abril 2004-marzo 2005.

\begin{tabular}{lcc}
\hline \multicolumn{1}{c}{ Levadura } & $\begin{array}{c}\text { Tiempo } \\
\text { (horas) }\end{array}$ & $\begin{array}{c}\text { Intervalo de } \\
\text { confianza al } \\
\mathbf{9 5 \%} \mathbf{( \pm )}\end{array}$ \\
\hline Kluyveromyces marxianus & $19 \mathrm{a}$ & 0 \\
Saccharomyces cerevisiae & $24 \mathrm{~b}$ & 2 \\
Candida Kefyr & $18 \mathrm{a}$ & 1 \\
\hline
\end{tabular}

$\mathrm{a}, \mathrm{b}$ Letras iguales indican que no se encontraron diferencias significativas $(\mathrm{p} \leq 0,05)$. 
prolongado implica un proceso de producción más extenso y como consecuencia, con mayores costos de operación que las otras dos levaduras (Stanbury y Whitaker 1987).

$K$. marxianus y $C$. kefyr no presentaron diferencias significativas $(p \geq 0,05)$ en cuanto al tiempo de fermentación. El presentar un ciclo de crecimiento de igual duración, implica que los costos de proceso serían muy similares para ambas levaduras, considerando únicamente el tiempo que tardaría cada ciclo de producción, sin embargo, se deben tomar en cuenta otros parámetros como la utilización de lactosa del medio, el contenido de proteína y productividad de cada una de las levaduras, para obtener conclusiones al respecto.

Al comparar el consumo de lactosa al final del proceso, tal como se presenta en el Cuadro 3, se observa que no hubo diferencias significativas en la cantidad de lactosa empleada por cada una de las levaduras hasta alcanzar la fase estacionaria, lo que indica que la hidrólisis de este azúcar, posiblemente ocurrió a una misma velocidad con cada una ellas.

Cuadro 3. Utilización de lactosa para el crecimiento de Kluyveromyces marxianus y Candida Kefyr en suero de queso. Costa Rica, abril 2004-marzo 2005.

\begin{tabular}{lcc}
\hline \multicolumn{1}{c}{ Levadura } & $\begin{array}{c}\text { Lactosa } \\
\text { utilizada }(\%)\end{array}$ & $\begin{array}{c}\text { Intervalo de } \\
\text { confianza al } \\
\mathbf{9 5 \%}(\mathbf{\pm})\end{array}$ \\
\hline Kluyveromyces marxianus & 79 & 0,7 \\
Candida Kefyr & 75 & 1,0 \\
\hline
\end{tabular}

* No hubo diferencias significativas al 95\% de confianza.

El tiempo de fermentación y la reducción de lactosa obtenidos para K. marxianus coinciden con los resultados de investigaciones en las que se utilizó esta misma especie. Según Belem et al. (1997), la levadura utilizada alcanzó la fase estacionaria a las $20 \mathrm{~h}$ de crecimiento y logró una reducción de la lactosa de un $80 \%$, durante su crecimiento en suero de queso suplementado. Respecto a lo anterior, es importante considerar que en el caso del presente estudio no se suplementó el suero y se obtuvo un resultado muy similar a estudios en los que sí se llevó a cabo. Esta situación da la posibilidad de evaluar el efecto de la suplementación sobre el tiempo y cantidad de biomasa generada, lo que podría mejorar el proceso. La adición de nutrientes al medio, como sales de amonio y extracto de levadura, puede tener un efecto beneficioso en la producción y actividad de la enzima ß-galactosidasa (De Bales y Castillo 1979).

Un aspecto que podría evaluarse para disminuir el tiempo de fermentación con $K$. marxianus y $C$. kefyr es el utilizar para la propagación de los cultivos, un medio que contenga lactosa, o el mismo suero a utilizar en la fermentación. Con lo anterior, se estaría realizando la activación del microorganismo sobre un medio con el mismo azúcar del que dispondrá en el medio de fermentación, con lo que se podría acortar la fase de adaptación (Fase Lag) del microorganismo (Chinappi y Sánchez 2000), y con ello el tiempo de la fermentación.

\section{Comparación de la productividad obtenida con las tres especies de levadura}

De las levaduras estudiadas, $K$. marxianus presentó una productividad significativamente mayor $(p \leq 0,05)$, tal como se observa en el Cuadro 4. Esto indica que la velocidad de formación de biomasa y con ello el crecimiento de esta levadura fue mayor, lo cual es deseable y necesario para un proceso de producción de proteína unicelular (Chacón 2004).

La menor productividad de biomasa de C. kefyr, respecto a la de $K$. marxianus, concuerda con lo

Cuadro 4. Productividad de las levaduras Kluyveromyces marxianus, Saccharomyces cerevisiae y Candida kefyr en la formación de biomasa microbiana en suero de queso. Costa Rica, abril 2004-marzo 2005.

\begin{tabular}{lcc}
\hline \multicolumn{1}{c}{ Levadura } & $\begin{array}{c}\text { Productividad } \\
(\mathbf{g} / \mathbf{l ~ h})\end{array}$ & $\begin{array}{c}\text { Intervalo de } \\
\text { confianza } \\
\text { al 95\% ( } \mathbf{})\end{array}$ \\
\hline Kluyveromyces marxianus & $0,22 \mathrm{a}$ & 0,02 \\
Saccharomyces cerevisiae & $0,14 \mathrm{~b}$ & 0,03 \\
Candida kefyr & $0,13 \mathrm{~b}$ & 0,01 \\
\hline
\end{tabular}

a, b Letras iguales indican que no se encontraron diferencias significativas $(\mathrm{p} \leq 0,05)$. 
reportado por Barroquio et al. (1981) y por Capoor y Singh (1985), quienes obtuvieron productividades menores utilizando una levadura Candida pseudotropicalis, en condiciones de crecimiento similares y utilizando suero de leche de composición similar a la del presente estudio. De acuerdo con la productividad obtenida con $C$. kefyr en comparación con la $K$. marxianus, se observa que ésta no se desarrolló de forma adecuada en el medio, ya que a pesar de que no presentó diferencia en el tiempo de fermentación respecto a $K$. marxianus, y la cantidad de lactosa utilizada fue muy similar (Cuadro 3), su velocidad de formación de biomasa fue menor, lo que implicaría costos más altos de producción por la extensión del tiempo del proceso.

En el caso de S. cerevisiae, el obtener una productividad de biomasa baja, puede relacionarse con la concentración inicial de azúcares en el medio de crecimiento. Una vez realizada la hidrólisis, la cantidad de azúcar presente en el medio era de $20 \mathrm{~g} / \mathrm{l}$ de glucosa, este nivel de azúcar, aún en condiciones aeróbicas, puede causar que las levaduras de la especie $S$. cerevisiae se desarrollen por la vía de la fermentación más que la de la respiración, por causa del efecto Crabtree (Shuler y Kargi 1992). El nivel de azúcar crítico, a partir del cual se produce este efecto, va a depender de la especie y cepa de levadura con la que se trabaje, según Moo-Young et al. (1985), puede ocurrir a niveles de glucosa mayores a tres o hasta $30 \mathrm{~g} / \mathrm{l}$. El efecto Crabtree causa la disminución drástica de la generación de biomasa, debido a que la utilización del sustrato (lactosa) se desvía hacia la producción de etanol, lo que hace que la formación de biomasa se vea disminuida de forma considerable (Postma et al. 1989).

La generación etanol por parte de esta especie de levadura ha llevado a investigadores a evaluar la producción de etanol con cepas genéticamente modificadas en la búsqueda de rendimientos rentables (Domingues et al. 2001).

\section{Comparación del contenido proteico de la biomasa de las levaduras estudiadas}

Las levaduras evaluadas no presentaron diferencias significativas $(p=0,0665)$ en cuanto al contenido de proteína de la biomasa. Tal como se presenta en el Cuadro 5, los porcentajes de proteína variaron entre un 32\% y un $35 \%$. Estos resultados son similares a los reportados por Barroquio et al. (1981), quienes obtuvieron

ISSN: 1021-7444
Cuadro 5. Contenido de proteína de la biomasa microbiana obtenida a partir del crecimiento en suero de queso de las levaduras Kluyveromyces marxianus, Saccharomyces cerevisiae y Candida Kefyr. Costa Rica, abril 2004-marzo 2005

\begin{tabular}{lcc}
\hline \multicolumn{1}{c}{ Levadura } & $\begin{array}{c}\text { Contenido de } \\
\text { proteína }(\mathbf{\%} / \mathbf{m}) *\end{array}$ & $\begin{array}{c}\text { Intervalo de } \\
\text { confianza al } \\
\mathbf{9 5 \%}(\mathbf{\pm})\end{array}$ \\
\hline Kluyveromyces marxianus & 32 & 2 \\
Saccharomyces cerevisiae & 35 & 2 \\
Candida Kefyr & 34 & 1 \\
\hline
\end{tabular}

* No hay diferencias significativas al 0,05\%.

porcentajes de $31 \%$ y $38 \%$, durante el crecimiento en suero de leche de las levaduras Kluyveromyces fragilis y Candida pseudotropicalis, respectivamente.

Al comparar el contenido proteico de la biomasa de las levaduras evaluadas, con el valor indicado en la composición general de las levaduras éste fue bajo, ya que en promedio el porcentaje de proteína es de alrededor de 45-55\% (Reed y Pepler 1983). Un menor contenido proteico se puede relacionar con características propias de las levaduras bajo estudio, así como el efecto de parámetros operativos y de la composición del medio de cultivo, como en el caso del presente estudio que no fue suplementado (Chinappi y Sánchez 2000). Diversos estudios demuestran que en medios de cultivo en los cuales se ha limitado la adición de nitrógeno, las levaduras presentan un contenido de proteína menor que las crecidas en un medio suplementado con este compuesto (Aiba et al. 1973). En el presente estudio, las levaduras fueron evaluadas utilizando un medio de crecimiento simple, con el fin de determinar la producción de proteína bajo las mismas condiciones y empleando un sustrato menos costoso.

\section{Selección del microorganismo para producción de proteína unicelular a partir de suero de queso}

Para la elección de un microorganismo a utilizarse en la producción de proteína unicelular, se tomaron en cuenta aspectos de índole económico y práctico, que podrían dar ventajas a utilizar un microorganismo u otro.

En un proceso por lotes o batch, el ciclo de crecimiento debe ser tan corto como sea posible, para

AGRONOMÍA MESOAMERICANA 17(2): 151-160. 2006 
obtener el mayor rendimiento y utilización del equipo (Stanbury y Whitaker 1987), esto implica una reducción de los costos de operación, que incluyen la energía, gastos generales, costos de mano de obra y la capacidad del sistema (Rojas 1995). Las levaduras K. marxianus y $C$. kefyr presentan esta ventaja respecto a $S$. cerevisiae al presentar un menor tiempo de fermentación.

A pesar de que las levaduras $K$. marxianus y $C$. kefyr presentaron un tiempo similar, es importante considerar que la productividad de biomasa de $K$. marxianus fue superior, tanto a la obtenida para $C$. kefyr como a la de $S$. cerevisiae. Una mayor velocidad de formación de biomasa da ventajas a su utilización en la obtención del producto.

Las levaduras $K$. marxianus y $S$. cerevisiae, son consideradas como organismos GRAS (Generalmente reconocida como segura) según la FDA (Liti et al. 2001). La cepa de $C$. kefyr empleada en esta investigación, a pesar de que es utilizada en diversas investigaciones y que no se ha encontrado que sea patógena (ATCC 2004), no ha sido aceptada como segura para ser utilizada en alimentación animal ni humana, por lo que sería necesario realizar un proceso de investigación previo a la utilización de su biomasa a nivel industrial.

El utilizar la levadura $S$. cerevisiae, implica un tiempo de fermentación más prolongado y una menor productividad. La hidrólisis enzimática de la lactosa del suero requiere la utilización de enzima libre, lo cual implica mayores costos económicos, tanto por la compra de los preparados enzimáticos, como por el tiempo y funcionamiento de equipos durante la hidrólisis.

Considerando los aspectos analizados anteriormente, la especie $K$. marxianus presentó mayores ventajas para ser utilizada en la producción de proteína unicelular en suero de leche, que las levaduras $C$. kefyr y $S$. cerevisiae. El comparar las tres levaduras bajo las mismas condiciones ambientales y sin la suplementación del suero, permitió determinar cómo $K$. marxiuanus se desarrolló de forma más eficiente en el medio. En caso de utilizarse esta levadura para el tratamiento del suero de queso en un sistema por lotes, presentaría un tiempo de fermentación menor, que resultaría en una ventaja económica y práctica, y una mayor productividad, que aseguraría una mayor velocidad en la generación del producto de interés.

Para la aplicación de esta levadura en la industria nacional, sería recomendable simplificar las condiciones del proceso. Para esto sería necesario, en siguientes investigaciones, optimizar el proceso a escala industrial realizando ciertas modificaciones, una de ellas sería el empleo de tanques de fermentación a temperatura ambiente. Además, sería necesario incorporar el proceso de forma paralela a la línea de producción de la planta, de manera que se utilice el producto final (biomasa) para la alimentación del ganado, dándole valor agregado a un desecho que generan las industrias lácteas.

\section{LITERATURA CITADA}

AIBA, S.; HUMPHREY, A.; MILLIS, N. 1973. Biochemical engineering. New York, USA. Academic Press. p. 259.

ALAIS, C. 1996. Ciencia de la leche: principios de técnica lechera. México, DF. Editorial CECSA. p. 56.

AOAC (Association of Official Analitical Chemists). 1999. Official methods of analysis. 15 ed. Washington D.C. Versión CD-ROM.

AOAC (Association of Official Analitical Chemists). 2000. Official methods of analysis. 16 ed. Washington D.C. Versión CD-ROM.

ATCC (American Type Culture Collection). 2004. Fungi, yeast and yeast generis stock (en línea). Manassas, USA. Consultado 2 mar. 2004. Disponible en: http:// www.atcc.com

BARROQUIO, V.; SILVERIO., L.; REVILLEZA, R.; FERNANDEZ, W. 1981. Production of protein-rich animal feed supplement from cheese whey. Milchwissenschaft 36(4): 209-211.

BELEM, M.; GIBBS, B.; LEE, B. 1997. Enzymatic production of Ribonucleotides from autolysates of Kluyveromyces marxianus grown on whey. Journal of Food Science 62(4): 851-857.

BU'LOCK, J.; KRISTIANSEN, B. 1991. Biotecnología básica. Zaragoza, España. Editorial Acribia. p. 285-307.

CAPOOR, A.; SINGH, K. 1985. Fermentation of whey by lactose utilization yeast for S.C.P production and B.O.D reduction. Diary Science 38 (1): 15-17.

CHACÓN, A. 2004. Perspectivas actuales de la proteína unicelular (SCP) en la agricultura y la industria. Agronomía Mesoamericana 15(1): 93-106.

CHAN, Y. 2001. Utilización de banano de rechazo para la generación de ácido láctico a través de un proceso de fermentación por lote. Tesis Lic. Tecnología de Alimentos. Costa Rica, Universidad de Costa Rica. p. 5-35.

AGRONOMÍA MESOAMERICANA 17(2): 151-160. 2006 
CHINAPPI, I.; SÁNCHEZ, J. 2000. Producción de biomasa de Kluyveromyces fragilis en suero de leche desproteinizado. Acta Científica Venezolana 51: 223-230.

CNP (Consejo Nacional de Producción). 1999. Dirección de mercadeo y agroindustria área desarrollo producto (en línea). Disponible en: ww.mercanet.cnp.go.cr/ Desarrollo_Agroid/documentospdf/Queso_FTP.pdf.

CNP (Consejo Nacional de Producción). 2001. Programa de lácteos: "Mejoramiento de la sanidad y calidad del queso fresco elaborado en las queserías artesanales y rurales de Costa Rica”. San José. 150 p.

DE BALES, S.; CASTILLO, F. 1979. Production of lactase from Candida pseudotropicales grown in whey. Applied and Enviromental Microbiology 37(6): 1201-1205.

DOMINGUES, L.; LIMA, N.; TEIXEIRA, J. 2001. Alcohol production from cheese whey permeate using genetically modified flocculent yeast cells. Biotechnology \& Bioengeneering 72(5): 507-514.

DURÁN, N. 1989. Bioconversion to single cell protein: recovery of lignocellulosic materials to produce human food as integrated process. Alimentos (Chile) 14 (4): 39-50.

GHALY, A.; KAMAL, M. 2004. Submerged yeast fermentation of acid cheese whey for protein production and pollution potencial reduction. Water Research 38: 631-644.

GRBA, S.; STEHLIK, T.; STANZER, N.; VAHÈIÆ, N.; SKRLIN, A. 2002. Selection of yeast strain of Kluyveromyces marxianus for alcohol and biomass production on whey. Chemical and Biochemical Engineering 16 (1): 13-16.

HERNÁNDEZ, E.; MAZA, E.; LOZANO, N. 1980. Producción de proteína unicelular mediante cultivo continuo de levadura en suero de leche desproteinizado. Revista de la Facultad de Agronomía (Venezuela) 5(2): 468-477.

INCHAURRONDO, V.; YANTORNO, O.; VOGET, C. 1994. Yeast growth and B-galatosidase production during bacth cultures in lactose-limited syntethic medium. Process Biochemistry 29: 47-54.

LEE, B. 1996. Fundamentos de biotecnología de los alimentos. Zaragoza, España. Editorial Acribia. p. 77-89.

LITI, G.; WARDROP, F.; CARDINALI,G.; MARTINI, A.; WALKER, G. 2001. Differential responses to antimycin A and expressions of the Crabtree effect in selected Klyuveromyces spp. Annals of Microbiology 51: 235-243.

MINISTERIO DE SALUD. 1997. Reglamento de reuso y vertido de aguas residuales. Decreto26042-S-MINAE, San José. p. 75.

ISSN: 1021-7444
MITTAL, G. 1992. Food biotechnology: techniques and applications. Pennsylvania, USA. Technomics Publishing Company. p. 245-305.

MOO-YOUNG, M.; BLANCH, H.; DREW, S.; WANG, D. 1985. Comprehensive biotecnology. v 3. Oxford, USA. Pergamon Press. p. 547-561.

POSTMA, E.; VERDUYN, C.; SCHEFFERS, W.; VAN DIJKEN, J. 1989. Enzymic analysis of the crabtree effect in glucose-limited chemostat cultures of Saccharomyces cerevisiae. Applied and Enviromental Microbiology 55(2): 468-477.

QUINTERO, H.; RODRÍGUEZ, M.; PÁEZ, G. 2001. Producción continua de proteína microbiana $(K$. fragilis) a partir de suero de leche. Revista de la Facultad de Ciencias Veterinarias de la Universidad de Zulia (Venezuela) 11(2): 87- 94.

RAJOKA, M.; KHAN, S.; SHAHID, R. 2003. Kinetics and regulation studies of the production of $\beta$ Galactosidase from Kluyveromyces marxianus grown on different substrates. Food Technology and Biotechnology 41(4): 315-320.

REED, G.; PEPLER, H. 1983. Industrial microbiology. Conneticutt, USA. AVI Publishing Company, INC. p. 541-581.

REVILLA, A. 1985. Tecnología de la leche. Guatemala. IICA. p. 15.

ROJAS, A. 1995. Obtención de proteína unicelular a partir de residuos de destilería. Tesis Lic. Química. Costa Rica, Universidad de Costa Rica. p.7-21.

ROSE, A. 1979. Economic microbiology: Microbial Biomass. v. 4. London. Academy Press. p. 208-256.

SALAZAR, N. 1999. Establecimiento de las condiciones adecuadas para la fabricación de suero en polvo a partir del suero producido en la elaboración del queso tipo Turrialba mediante la técnica de secado por atomización. Tesis Lic. Tecnología de Alimentos. Costa Rica, Universidad de Costa Rica. p. 5-25.

SCRAGG, M. 2000. Biotecnología para ingenieros: Sistemas biológicos en procesos tecnológicos. México, D.F. Editorial Limusa. p. 252.

SHULER, M.; KARGI, F. 1992. Bioprocess engineering: Basics concepts. New Jersey, USA. Editorial Prentice Hall. p. 25.

STANBURY, P.; WHITAKER, A. 1987. Principles of fermentation technology. Pergamon Press, New York. p.17-53.

WARD, O. 1991. Biotecnología de la fermentación. Zaragoza, España. Editorial Acribia. p. 5-127.

AGRONOMÍA MESOAMERICANA 17(2): 151-160. 2006 